DOI: 10.2478/awutm-2013-0001<smiles>CC1=C2CC2C1</smiles>

VERSITA
Analele Universităţii de Vest,

Timişoara

Seria Matematică - Informatică

LI, 1, (2013), 3- 24

\title{
Particle Swarm Clustering Optimization - a novel Swarm Intelligence approach to Global Optimization
}

\author{
George Anescu
}

\begin{abstract}
Clustering optimization methods for continuous numerical multivariable functions have been used for increasing the efficiency in the selection of the start points in multi-start global optimization methods. Methods of this kind usually have three steps: (1) sampling points in the search domain, (2) transforming the sampled points in order to obtain points grouped in neighbourhoods of local optima, (3) using a clustering technique to identify the clusters. After the clusters are successfully identified, the set of local optima (and from it the global optimum) can be easily determined by applying a local optimization method for each cluster. The novel Particle Swarm Clustering Optimization (PSCO) method proposed in this paper is concerned with simultaneous integration of steps (1), (2) and (3) from the classical clustering optimization methods by applying Swarm Intelligence (SI) techniques. Two existing SI methods provided inspiration in the design of the PSCO method: Particle Swarm Optimization (PSO) and Firefly Algorithm (FA).
\end{abstract}

AMS Subject Classification (2000). 68T20, 68W10, 90C26, 90C56, 90C59

Keywords. Clustering Optimization, Swarm Intelligence (SI), Continuous Global Optimization Problem (CGOP) Particle Swarm Optimization (PSO), Firefly Algorithm (FA), Fitness and Euclidean-distance Ratio (FER) 


\section{Introduction}

The present paper is proposing a novel global optimization method called Particle Swarm Clustering Optimization (PSCO) that integrates ideas from Clustering Optimization and Swarm Intelligence (SI). Clustering global optimization methods form a class of global optimization methods which as an important part include a cluster analysis technique with the purpose to recognize the regions of attraction by identifying the clusters corresponding to those regions. The clustering optimization methods can be viewed as a modified form of the standard multi-start procedure, which performs a local search from several points distributed over the entire search domain. A drawback of the multi-start procedure is that, when many starting points are used, the same local minimum may be identified several times, thereby leading to an inefficient global search. Clustering optimization methods attempt to avoid this inefficiency by carefully selecting the points at which the local search is initiated. The three main steps of clustering optimization methods are ([1]): (1) sampling points in the region of interest; (2) transforming the sampled points to obtain points grouped in neighbourhoods of local optima; and (3) using a clustering technique to identify the clusters which (hopefully) represent neighbourhoods of local optima. After the clusters are successfully identified, the set of local optima (and from it hopefully the global optimum) can be easily determined by applying a local optimization method for each identified cluster. The clustering problem could be a computationally difficult problem by itself, contributing to the computational complexity of the global optimization method. In the proposed PSCO method the three main steps of classical clustering optimization methods are integrated and performed simultaneously at each iteration step by applying Swarm Intelligence techniques, in this way the computationally difficult clustering problem being avoided. Swarm intelligence is the discipline that deals with natural and artificial systems composed of populations of simple agents interacting locally with one another and with their environment. The agents follow very simple rules, and although there is no centralized control structure dictating how individual agents should behave, local and random interactions between such agents lead to the emergence of "intelligent" global behaviour, unknown to the individual agents. Examples of SI global optimization methods based on rules observed in nature or on mathematical and physical principles are: Ant Colony Optimization (ACO) ([2]), Particle Swarm Optimization (PSO) ([3], [4], [5], [6]), Firefly Algorithm (FA) ([7], [8]), River Formation Dynamics (RFD) ([9]), Stochastic Diffusion Search (SDS), Gravitational Search Algorithm (GSA) ([10]), Intelligent Water Drops (IWD) ([11]), Charged System 
Search (CSS) ([12]), etc.

\section{The Continuous Global Optimization Problem (CGOP)}

The general Continuous Global Optimization Problem (CGOP) is formulated as:

$$
\begin{array}{ll}
\operatorname{minimize} & f(\mathbf{x}) \\
\text { subject to } & \mathbf{x} \in D
\end{array}
$$

with

$$
\begin{aligned}
D=\{\mathbf{x}: \mathbf{l} \leq \mathbf{x} \leq \mathbf{u} ; & g_{i}(\mathbf{x}) \leq 0, \quad i=1, \ldots, G \\
& \left.h_{j}(\mathbf{x})=0, \quad j=1, \ldots, H\right\}
\end{aligned}
$$

where $\mathbf{x} \in \mathbb{R}^{n}$ is a real $n$-vector of decision variables, $f: \mathbb{R}^{n} \rightarrow \mathbb{R}$ is the continuous objective function, $D \subset \mathbb{R}^{n}$ is the non-empty set of feasible decisions (a proper subset of $\mathbb{R}^{n}$ ), $\mathbf{l}$ and $\mathbf{u}$ are explicit, finite (component-wise) lower and upper bounds on $\mathbf{x}, g_{i}: \mathbb{R}^{n} \rightarrow \mathbb{R}, i=1, \ldots, G$ is a finite collection of continuous inequality constraint functions, and $h_{j}: \mathbb{R}^{n} \rightarrow \mathbb{R}, j=1, \ldots, H$ is a finite collection of continuous equality constraint functions. Although each equality constraints can be equivalently formulated as two inequality constraints $(h(\mathbf{x})=0$ is equivalent to $h(\mathbf{x}) \leq 0$ and $-h(\mathbf{x}) \leq 0)$ and therefore $D$ can be defined even more generally only with inequality constraint functions ([13]), we prefer (2.2) because the equality constraints and the inequality constraints will be treated numerically in different manners by the PSCO method. We don't make any other additional assumptions on the CGOP problem and assume that we cannot get any additional knowledge about the collections of real continuous functions defined, in this way treating the CGOP problem as a black box, i.e. for any point $\mathbf{x}$ in the boxed domain $\{\mathbf{x}: \mathbf{l} \leq \mathbf{x} \leq \mathbf{u}\}$ we assume that we are able to calculate the values of the functions $f(\mathbf{x}), g_{i}(\mathbf{x}), i=1, \ldots, G, h_{j}(\mathbf{x}), j=1, \ldots, H$, but nothing more. One advantage of the SI optimization methods is that they are derivative free, in this way being effective regardless of the nature of the objective function and constraints. In the above listed general analytical conditions, the classical theorem of Weierstrass guarantees that the optimal solution set of the CGOP is non-empty. At the same time, without further specific structural assumptions, (2.1) with (2.2) can be a very difficult problem. For 
instance, $D$ could be disconnected, and even some of its components could be non-convex; furthermore, $f$ could be highly multimodal. Hence, there may exist an unknown number of global and local solutions to (2.1) with (2.2). Generally speaking there are two types of methods for approaching the CGOP problem (2.1), (2.2) (for a general survey see [13], [14], [15]): (1) Exact methods; (2) Heuristic methods. The heuristic methods, as a rule, do not have strict convergence guarantees; however, in many cases, they may offer practical tools to handle models that are (currently or forever) out of the reach of a theoretically correct, rigorous methodology. The two major components of any heuristic algorithm are: selection of the best solutions and randomization. The selection of the best ensures that the solutions will converge to the optimum, while the randomization avoids the trapping of the solutions in a local optimum, at the same time increasing the diversity of the solutions. A good combination of the two components will usually ensure that the global optimum is achievable.

\section{Ideas from the PSO methods}

PSO is a method for performing numerical optimization without explicit knowledge of the derivatives of the problem to be optimized (black box, derivative free approach). PSO is originally attributed to Kennedy, Eberhart and Shi (1995 - 1998) ([4], [5]) and was first intended for simulating social behaviour. The algorithm was simplified and it was observed to be performing well in optimization problems. The standard (canonical) PSO model is developed based on the following assumptions: (1) When one particle locates an optimum of the objective function, it instantaneously transmits the information to all the other particles; (2) All the other particles gravitate to the optimum of the objective function, but not directly and by simulating a random search in the search space; (3) There is a component of each particle's own independent thinking and past memory. In the context of multivariable optimization, the swarm is assumed to be of a specified fixed size $N$ with each particle located initially at random locations in the multidimensional search space. Each particle is assumed to have three characteristics: a position $\mathbf{x}_{i}$, a velocity $\mathbf{v}_{i}$ and a best personal position $\mathbf{x}_{i}^{\text {best }}, i=1, \ldots, N$. The personal bests are forming the memory-swarm to provide a stable network retaining the best positions (based on objective function value) found so far by the population, while the current positions act as parts of an explorer-swarm to explore broadly around the search space. The global best position, denoted by $\mathbf{x}^{g b e s t}$, represents the best particle position found so far. At each iteration 
step the velocities are updated and the particles are moved to new positions. The updates of the velocities are calculated as the previous values plus the contribution of the interaction of the particles with their own best, and plus the contribution of the interaction of the particles with the global best:

$$
\begin{aligned}
\mathbf{v}_{i}(k) & =\theta(k) \mathbf{v}_{i}(k-1)+c_{1} r_{1}\left[\mathbf{x}_{i}^{\text {best }}(k-1)-\mathbf{x}_{i}(k-1)\right]+ \\
& +c_{2} r_{2}\left[\mathbf{x}^{\text {gbest }}(k-1)-\mathbf{x}_{i}(k-1)\right], \quad i=1, \ldots, N
\end{aligned}
$$

where $c_{1}$ and $c_{2}$ are constants called acceleration coefficients, $\theta(k)$ is an inertia factor, $r_{1}$ and $r_{2}$ are random numbers uniformly distributed in the range [0,1), and $k$ is the current iteration index. The new positions are determined from the previous positions and the new velocities according to:

$$
\mathbf{x}_{i}(k)=\mathbf{x}_{i}(k-1)+\mathbf{v}_{i}(k), \quad i=1, \ldots, N
$$

As such, gradually over many iterations, the particles go to the target, the global optimum of the objective function. From the presented standard PSO model we retain for the design of our PSCO method the valuable ideas of using a memory-swarm (besides the explorer-swarm) and taking into account the interactions between the explorer-swarm and the memory-swarm. There are many other more advanced PSO models, some of them capable to handle multimodal objective functions with the purpose to find all or the most important modes of the objective function (given that the population size $N$ is sufficiently large). One such model is FER-PSO ([16], [17]) where, instead of using a single global best, each particle is attracted towards a fittest-and-closest neighbourhood point which is identified via computing its FER (Fitness and Euclidean-distance Ratio) value:

$$
F E R_{i}=\alpha \max _{1 \leq j \leq N, j \neq i} \frac{f\left(\mathbf{x}_{i}^{\text {best }}\right)-f\left(\mathbf{x}_{j}^{\text {best }}\right)}{\left\|\mathbf{x}_{i}^{\text {best }}-\mathbf{x}_{j}^{\text {best }}\right\|_{2}}, i=1, \ldots, N
$$

where $\alpha$ is a scaling factor:

$$
\alpha=\frac{\|\mathbf{u}-\mathbf{l}\|_{2}}{f\left(\mathbf{x}^{\text {gbest }}\right)-f\left(\mathbf{x}^{\text {gworst }}\right)}
$$

and $\mathbf{x}^{\text {gworst }}$ is the position of the worst positioned particle. Notice that in the estimation of the FER value of a particle the memory-swarm is used. The idea of using the FER values is also valuable and it will be generalized and used in the design of the novel PSCO method. 


\section{Ideas from the Firefly Algorithm (FA)}

The FA algorithm was developed by Xin-She Yang at Cambridge University in 2007 ([7], [8]) and is based on the following simplifications: (1) All fireflies are unisex, so that one firefly will be attracted to all other fireflies; (2) Attractiveness is proportional to their brightness, and for any two fireflies, the less bright one will be attracted (and thus move) to the brighter one; however, the brightness can decrease as their distance increases; (3) If there are no fireflies brighter than a given firefly, it will move randomly. In the firefly algorithm, there are two important issues: the variation of light intensity and the formulation of the attractiveness. For simplicity, it is always assumed that the attractiveness of a firefly is determined by its brightness which in turn is associated with the encoded objective function. However, the attractiveness $\beta$ is relative, it should be seen in the eyes of the beholder or judged by the other fireflies. Thus, it will vary with the distance $r_{i, j}$ between firefly $i$ and firefly $j$. In addition, light intensity decreases with the distance from its source, and light is also absorbed in the media, so we should allow the attractiveness to vary with the degree of absorption $\gamma$. A firefly's attractiveness is proportional to the light intensity seen by adjacent fireflies. In practice the following formula, having the above characteristics, is used with good results:

$$
\beta=\beta_{0} e^{-\gamma r^{2}}
$$

where $\beta_{0}$ is the attractiveness at $r=0$. With the absorption coefficient $\gamma$ well tuned, FA is able to find the global minimum as well as the local minimums simultaneously. A valuable idea from the FA method used in the design of the PSCO method, is that of mutual interactions (everybody with everybody) of the fireflies.

Notice that the FA method is not using at all the memory-swarm. At each iteration step $k$ the new positions of the fireflies are calculated according to the algorithm:

$$
\begin{aligned}
& \text { for } i=1 \text { to } N \text { do } \\
& \text { for } l=1 \text { to } N \text { do } \\
& \quad \text { if } f\left(\mathbf{x}_{l}\right)<f\left(\mathbf{x}_{i}\right) \text { move firefly } i \text { towards firefly } l \text { according } \\
& \quad \text { to the formula: } \\
& \quad \mathbf{x}_{i}(k)=\mathbf{x}_{i}(k)+\beta_{i, l}\left[\mathbf{x}_{l}(k)-\mathbf{x}_{i}(k-1)\right]+\alpha(k)\left(\mathbf{r n d}_{i}-\mathbf{0 . 5}\right)
\end{aligned}
$$




\section{end for $i$}

where the positions of the fireflies at iteration $k$ are initialized to the last values at iteration $k-1, \mathbf{x}_{i}(k)=\mathbf{x}_{i}(k-1), i=1, \ldots, N$. The usual formula for the attraction is:

$$
\beta_{i, l}=\beta_{0} e^{-\gamma r_{i, l}^{2}}, \quad r_{i, l}=\left\|\mathbf{x}_{l}(k)-\mathbf{x}_{i}(k-1)\right\|_{2}
$$

with $\beta_{0}$ a tuned parameter. We can see that for large distances between the fireflies the $\beta_{i, l}$ coefficients become insignificant and the attraction is very small. The need of an mechanism to reduce the intensity of interaction between particles with the distance is a valuable idea used in the design of the PSCO method. The third term is a randomization contribution with $\alpha(k)$ being a randomization parameter, and $\mathbf{r n d}_{i}$ a vector of random components in the interval $[0,1)$. The randomization parameter $\alpha(k)$ is dependent on the iteration index according to the formula:

$$
\alpha(k)=\text { rate } \times \alpha(k-1)
$$

where rate is the decrease rate calculated so that when the maximum iteration counter value iter $_{\max }$ is attained the randomization contribution has the magnitude of the required precision $\epsilon$. In this way the movements of the fireflies are frozen within the bounds of the required precision at the end of the algorithm. We will retain two other valuable ideas from the FA method for use in the design of our PSCO method: the rigorous control of the number of iterations iter $_{\max }$ needed for the convergence of the method (pre-calculated based on the required precision $\epsilon$ and the decrease rate rate) and the freezing of the movement of the particles within the bounds of the required precision $\epsilon$ at the end of the algorithm.

\section{The PSCO Algorithm}

For simplification we will treat first the Box Constrained Continuous Global Optimization Problem (without constraints). The constrained case of CGOP will be treated later in section 7 . The simplified pseudo code of the PSCO algorithm is given below:

Step 1: input the optimization problem;

Step 2: input the PSCO method's parameters;

Step 3: do initializations for iteration index $k=0$; 
for $k=1$ to iter $_{\max }$ do

foreach sub-swarm in the list of current sub-swarms do foreach particle $p_{i}$ in the current sub-swarm do

Step 4: update the particle's $p_{i}$ explorer-swarm position $\mathbf{x}_{i}(k)$ based on the interaction with its own best current memory-swarm position $\mathbf{x}_{i}^{\text {best }}(k-1)$;

end for

foreach particle $p_{i}$ in the current sub-swarm do

foreach particle $p_{l}$ in the interaction list of particle $p_{i}$ do

Step 5: update the particle's $p_{i}$ explorer-swarm position $\mathbf{x}_{i}(k)$ based on the interaction with particle's $p_{l}$ current best memory-swarm position $\mathbf{x}_{l}^{\text {best }}(k-1)$;

end for $l$

end for $i$

foreach particle $p_{i}$ in the current sub-swarm do Step 6: update $p_{i}$ particle's interaction list based on distance comparisons with the double of the current radius of action;

Step 7: update the particle $p_{i}$ explorer-swarm position $\mathbf{x}_{i}(k)$ so that to avoid swarm explosion;

Step 8: add random contribution to particle $p_{i}$ explorer-swarm position $\mathbf{x}_{i}(k)$;

\section{end for $i$}

Step 9: separate the current sub-swarm in new sub-swarms if the separation conditions apply;

\section{end for}

Step 10: update the radius of action;

foreach sub-swarm in the list of sub-swarms do

foreach particle $p_{i}$ in the current sub-swarm do

Step 11: recalculate the objective function's values in the new calculated particle's sub-swarms position and update the current best position $\mathbf{x}_{i}^{\text {best }}(k)$ if conditions apply. Update the explorer-swarm's and memory-swarm's weight values;

\section{end for $i$}

\section{end for}

end for $k$

foreach sub-swarm in the list of sub-swarms do

Step 12: take the best particle in the current sub-swarm 
(cluster) as the starting point for the local optimization method;

\section{end for}

In the remaining of this section each step of the PSCO algorithm will be detailed and all the involved notions will be clarified.

Step 1: The dimension of the search space $n$ and the bounds of the constraining box $\mathbf{l}$ and $\mathbf{u}$ are given as input parameters. Also the objective function $f(\mathbf{x})$ is loaded and it is assumed that its value can be calculated for any point $\mathbf{x} \in D$. No other specific information about the objective function $f(\mathbf{x})$ is given (black box approach).

Step 2: The PSCO method's specific input parameters are:

$p$ - the power parameter of Minkovski distance;

$N_{\max }$ - the initial number of particles in the swarm;

$\epsilon$ - the method's precision (radius of the final clusters);

pow - the distance power parameter;

$\alpha_{v}$ - the volumetric decrease rate;

The PSCO method works with the Minkovski distance, a generalization of the usual Euclidian distance which is a particular case for $p=2$ :

$$
\|\mathbf{x}-\mathbf{y}\|_{p}=\left(\sum_{j=1}^{n}\left|x_{j}-y_{j}\right|^{p}\right)^{\frac{1}{p}}, p \in \mathbb{R}, p>0, \mathbf{x} \in \mathbb{R}^{n}, \quad \mathbf{y} \in \mathbb{R}^{n}
$$

The precision parameter $\epsilon$ is related to our intuitive expectation of the size of the basins of attraction associated with the local optima. Local optima with small basins of attraction (having the radius less than $\epsilon$ ) may not be located during the clustering phase (there will be no distinct clusters associated to them), but they may be located later during the local optimization phase.

Step 3: Some initialization is necessary before starting the iterative process. First we transform the constraining box in the normalized box $[0,1]^{n}$ with the linear transforms:

$$
\mathbf{x}_{j}^{\prime}=\frac{\mathbf{x}_{j}-\mathbf{l}_{j}}{\mathbf{u}_{j}-\mathbf{l}_{j}}, j=1, \ldots n
$$

Each time we need to calculate the objective function $f(\mathbf{x})$ we will need to do the reverse transforms: 


$$
\mathbf{x}_{j}=\mathbf{l}_{j}+\left(\mathbf{u}_{j}-\mathbf{l}_{j}\right) \mathbf{x}_{j}^{\prime}, \quad j=1, \ldots n
$$

We will continue to use during the presentation the $\mathbf{x}$ notation instead of $\mathbf{x}^{\prime}$, just for simplicity, but we will keep in mind that some transforms are taking place in the background. A central idea in the PSCO method is to use a distance quantity called the radius of action $r_{a}$ in order to limits the interactions between particles. As a rule two particles will interact only if the distance between their best memory-swarm positions is less than the radius of action $r_{a}$. The radius at action is decreased at each iteration with a radial decrease rate. At initialization $r_{a}$ should have a large value so that almost all particles are able to mutually interact (everybody with everybody). A good choice for the initial value $r_{a}(0)$ is the radius of the Minkovski hyper-ball with unit volume (i.e. the same volume as the normalized box). The Minkovski hyper-ball with generic radius $r$ is defined by:

$$
B_{n, p}(r)=\left\{\mathbf{x} \in \mathbb{R}^{n}:\|\mathbf{x}\|_{p} \leq r\right\}
$$

If $V($.$) is the notation of the volume function then it can be demonstrated$ that $r_{a}$ is given by:

$$
r_{a}(0)=\frac{1}{V\left(B_{n, p}(1)\right)^{\frac{1}{n}}}
$$

At the end of the iterative process all the particles should be frozen within the bounds of a Minkovski hyper-ball with radius $\epsilon$. Based on this requirement and having $r_{a}(0)$ we can calculate the number of iterations:

$$
\text { iter }_{\text {max }}=\left\lfloor\frac{\log \left(\frac{\epsilon}{r_{a}(0)}\right)}{\log \alpha_{r}}\right\rfloor+1=\left\lfloor\frac{n \log \left(\frac{\epsilon}{r_{a}(0)}\right)}{\log \alpha_{v}}\right\rfloor+1
$$

where $\alpha_{r}$ is the radial decrease rate calculated based on the volumetric decrease rate $\alpha_{v}$ :

$$
\alpha_{r}=\left(\alpha_{v}\right)^{\frac{1}{n}}
$$

As we can see, the number of iterations can be pre-calculated and it is linear with the dimension $n$ of the search space. The particles are organized in a list of sub-swarms, but initially there is just one sub-swarm formed by all the initial $N_{\max }$ particles. We initialize the particle's $p_{i}$ explorer-swarm position $\mathbf{x}_{i}(0), i=1, \ldots N_{\max }$, randomly in the unit box $[0,1]^{n}, \mathbf{x}_{i, j}(0)=$ $\operatorname{rand}(), j=1, \ldots n$, where the function $\operatorname{rand}()$ generates pseudo-random numbers in the interval $[0,1)$. Initially the memory-swarm is identical to the 
explorer-swarm $\mathbf{x}_{i}^{\text {best }}(0)=\mathbf{x}_{i}(0), \quad i=1, \ldots N_{\max }$. Also each particle has an associated interaction list which is initialized to contain all the other particles. We also calculate the values of the objective function in the current explorerswarm positions and based on them we initialize the weights associated to the current explorer-swarm positions and the current best (memory-swarm) positions:

$$
\mathbf{w}_{i}^{\text {best }}(0)=\mathbf{w}_{i}(0)=\frac{v_{\max }-f\left(\mathbf{x}_{i}(0)\right)}{v_{\max }-v_{\min }}, i=1, \ldots N_{\max }
$$

where

$$
\begin{gathered}
v_{\text {min }}=\min _{1 \leq i \leq N_{\max }} f\left(\mathbf{x}_{i}(0)\right), \\
v_{\max }=\max _{1 \leq i \leq N_{\max }} f\left(\mathbf{x}_{i}(0)\right)
\end{gathered}
$$

Step 4: A particle is interacting to its own best position only if the distance between its explorer-swarm position and its memory-swarm position is less than the current radius of action $r_{a}(k-1)$ :

$$
\left\|\mathbf{x}_{i}(k-1)-\mathbf{x}_{i}^{\text {best }}(k-1)\right\|_{p} \leq r_{a}(k-1)
$$

The new position is calculated on each component by:

$$
\mathbf{x}_{i, j}(k)=\mathbf{x}_{i, j}(k-1)+f_{i, i}\left(\mathbf{x}_{i, j}^{\text {best }}(k-1)-\mathbf{x}_{i, j}(k-1)\right), j=1, \ldots n
$$

where the factor $f_{i, i}$ is given by:

$$
f_{i, i}=\frac{\mathbf{w}_{i}^{\text {best }}(k-1)-\mathbf{w}_{i}(k-1)}{\left\|\mathbf{x}_{i}(k-1)-\mathbf{x}_{i}^{\text {best }}(k-1)\right\|_{p}^{\text {pow }}}
$$

If condition (5.10) is not satisfied then the explorer-swarm position is reset to the memory-swarm position $\mathbf{x}_{i}(k)=\mathbf{x}_{i}^{\text {best }}(k-1)$. We never allow a particle to move at a distance greater than the current radius of action from its own memory-swarm position.

Step 5: The interactions are between the explorer-swarm and the memoryswarm, i.e. the interaction between the particle $p_{i}$ and the particle $p_{l}$ is between the positions $\mathbf{x}_{i}(k-1)$ and $\mathbf{x}_{l}^{\text {best }}(k-1)$ and the positions $\mathbf{x}_{i}^{\text {best }}(k-1)$ and $\mathbf{x}_{l}(k-1)$. The interaction takes place only if the distance between the memory-swarm positions of the particles $p_{i}$ and $p_{l}$ is less than the current radius of action $r_{a}(k-1)$ : 


$$
\left\|\mathbf{x}_{i}^{\text {best }}(k-1)-\mathbf{x}_{l}^{\text {best }}(k-1)\right\|_{p} \leq r_{a}(k-1)
$$

The new position is calculated on each component by:

$$
\mathbf{x}_{i, j}(k)=\mathbf{x}_{i, j}(k)+f_{i, l}\left(\mathbf{x}_{l, j}^{\text {best }}(k-1)-\mathbf{x}_{i, j}(k-1)\right), j=1, \ldots n
$$

where the factor $f_{i, l}$ is given by:

$$
f_{i, l}=\frac{\mathbf{w}_{l}^{\text {best }}(k-1)-\mathbf{w}_{i}(k-1)}{\left\|\mathbf{x}_{i}(k-1)-\mathbf{x}_{l}^{\text {best }}(k-1)\right\|_{p}^{\text {pow }}}
$$

The factors $f_{i, l}$ (and also the factors $f_{i, i}$ defined at Step 4 ) are generalizations of the Fitness and Euclidean-distance Ratio (FER) values mentioned in Section 3 above. They allow to quantitatively describe the strength of the interactions. Note that the factors $f_{i, l}$ could also be negative, in which case we have repulsion instead of attraction.

Step 6: All the particles $p_{l}$ for which the memory-swarm positions are relative to the memory-swarm position of particle $p_{i}$ at a distance greater than the double of the radius of action:

$$
\left\|\mathbf{x}_{i}^{\text {best }}(k-1)-\mathbf{x}_{l}^{\text {best }}(k-1)\right\|_{p}>2 r_{a}(k-1)
$$

are eliminated from the interaction list of particle $p_{i}$. This rule is reciprocal, i.e. $p_{i}$ will also be eliminated from the interaction list of $p_{l}$

Step 7: If the particles move to a large distance from their previous explorerswarm positions we have the phenomenon of swarm explosion which has a harmful effect on the convergence of the PSCO method and should be avoided. Therefore we limit the distance of movement to the current value of the radius of action $r_{a}(k-1)$, but we keep the direction of movement $\mathbf{x}_{i}(k)-\mathbf{x}_{i}(k-1)$.

Step 8: We add random contributions to the calculated particle positions in order to allow the particles to stochastically search for better positions in the search space:

$$
\mathbf{x}_{i, j}(k)=\mathbf{x}_{i, j}(k)+r_{a}(k-1)(\operatorname{rand}()-0.5), \quad j=1, \ldots n
$$

where the function $\operatorname{rand}()$ generates pseudo-random numbers in the interval $[0,1)$. The random contributions are proportional to the current radius of 
action, in this way ensuring that at the end of the iterative process the particles are frozen within the required precision limits.

Step 9: We can view a sub-swarm as a graph with the sub-swarm's particles being the vertices of the graph and the edges being formed by the following rule: a pair of vertices $p_{i}, p_{l}$ are considered connected if the following condition is satisfied:

$$
\left\|\mathbf{x}_{i}^{\text {best }}(k-1)-\mathbf{x}_{l}^{\text {best }}(k-1)\right\|_{p} \leq 2 r_{a}(k-1)
$$

Based on this definition of the graph edge and applying some algorithms from the graph theory (Depth-First Search for example) we can separate the graph in independent connected components which are considered new subswarms. After the separation each sub-swarm evolves independently from the other sub-swarms. We expect that each sub-swarm converges to a different local optimum by independently solving an optimization sub-problem, and here we can see a great opportunity to increase the efficiency of the method through parallel computing, by treating each sub-swarm in a parallel execution thread.

Step 10: The radius of action is simply updated by:

$$
r_{a}(k)=\alpha_{r} r_{a}(k-1)
$$

Step 11: We recalculate the objective function's values in the current explorerswarm positions and based on them we update the memory-swarm. If $f\left(\mathbf{x}_{i}(k)\right)<f\left(\mathbf{x}_{i}^{\text {best }}(k-1)\right)$ then we update $\mathbf{x}_{i}^{\text {best }}(k)=\mathbf{x}_{i}(k)$, otherwise we keep $\mathbf{x}_{i}^{\text {best }}(k)=\mathbf{x}_{i}^{\text {best }}(k-1)$. We recalculate the weights associated to the current explorer-swarm positions and the current best memory-swarm positions:

$$
\begin{aligned}
\mathbf{w}_{i}(k) & =\frac{v_{\max }-f\left(\mathbf{x}_{i}(k)\right)}{v_{\max }-v_{\min }} \\
\mathbf{w}_{i}^{\text {best }}(k) & =\frac{v_{\text {max }}-f\left(\mathbf{x}_{i}^{\text {best }}(k)\right)}{v_{\max }-v_{\min }}
\end{aligned}
$$

where

$$
\begin{aligned}
& v_{\text {min }}=\min _{i \in \text { current sub-swarm }} f\left(\mathbf{x}_{i}^{\text {best }}(k)\right), \\
& v_{\text {max }}=\max _{i \in \text { current sub-swarm }} f\left(\mathbf{x}_{i}(k)\right)
\end{aligned}
$$


Step 12: There is no need of any additional clustering analysis method, each sub-swarm in the list of sub-swarms representing a cluster. Any local optimization method can be applied, even other SI methods, but this time the local optimization method is starting from a constraining box much smaller than the one of the original problem. After running the local optimization method for all the obtained clusters we can make a list of the found local modes and from this list we can identify the best local mode which is considered the solution to the global optimization problem.

\section{Swarm Size Management}

As already mentioned, we view our CGOP problem as a black box, i.e. we don't have any specific information about the objective function or the constraints, we are only able to compute them. We don't know in advance if our objective function is unimodal or multimodal. Therefore, as a conservative rule we will start with a large number of particles $N_{\max }$ in the initial swarm. The problem is that if our objective function is unimodal and no separation occurs during the execution, then we will do all the processing with a large number of particles. This is not advantageous from the computational efficiency perspective. Another problem is that if our objective function is multimodal, with a large number of modes, we will not be able to find too many modes due to the small number of separations that occur with a limited number of particles. Our purpose is to find as many modes as possible in this way increasing the probability to find the global mode. Definitely we need a self-adaptive procedure for decreasing or increasing the number of particles in sub-swarms as the particular optimization problem requires. There can be imagined many algorithms for this purpose, what is presented below is just a possibility which produced good practical results. For decreasing/increasing the number of particles in a sub-swarm we need to introduce a new method parameter, $N_{\min }$ which is the minimum number of particles a sub-swarm can have during the iterative process. We need to calculate a current number of particles that a sub-swarm should have in a manner dependent on the current iteration index, so that at the end of the iterative process this current number of particles is equal to $N_{\min }$. Also we don't want this number to decrease too abruptly at the beginning of the iterative process. Fortunately, in the PSCO method we know in advance the number of iterations iter $_{\max }$ (see (5.6)), which represents a great advantage. The following binomial formula serves very well to our purposes: 


$$
N_{\text {curr }}(i t)=N_{\max }-\left\lfloor\left(\frac{N_{\max }-N_{\min }}{i t e r_{\max }^{2}}\right) i t^{2}+0.5\right\rfloor
$$

where $i t$ is the current iteration index and $N_{\text {curr }}(i t)$ is the current number of particles a sub-swarm should have as a function of the current iteration index. In order to avoid the reduction of the number of particles to often we will apply a kind of hysteresis rule. We define a maximum value acceptable for the current size of a sub-swarm $N_{c u r r, \max }=N_{\text {curr }} / 0.9$ and we will reduce the number of particles in the sub swarm to $N_{\text {curr }}$ only if the size of the subswarm is higher than $N_{c u r r, m a x}$, taking care to not eliminate the best particle in the sub-swarm.

At separation, as a rule, we eliminate the sub-swarms that separate in only one isolated particle, considering such a separation as accidental. After a number of delay iterations during which the separated sub-swarm is checked for stability, the sub-swarm size is increased to $N_{\min }$ immediately. After that the size is increased gradually in each iteration toward $N_{\text {curr }}$ with a constant rate, for example the inverse of the volumetric rate $1 / \alpha_{v}$.

The swarm-management step can be inserted in the PSCO algorithm between Step 10 and Step 11.

\section{$7 \quad$ Treatment of Constraints}

One of the most popular method for treating the constraints in SI optimization methods is the exterior penalty function method. The main idea in the penalty function methods is to apply an unconstrained optimization techniques on a modified objective function obtained by adding a penalty term to the original objective function in order to increase the optimization objective for any constraint violation. With the notations from (2.1) and (2.2) the new objective function has usually the form:

$$
F(\mathbf{x} ; \mathbf{a}(k), \mathbf{b}(k))=f(\mathbf{x})+\sum_{i=1}^{G} \mathbf{a}_{i}(k) \max \left[0, g_{i}(\mathbf{x})\right]^{p}+\sum_{j=1}^{H} \mathbf{b}_{j}(k)\left|h_{j}(\mathbf{x})\right|^{p}
$$

where the vectors $\mathbf{a}(k), \mathbf{b}(k)$ represent the penalty parameters which are dependent on the iteration index $k$ and increase to very large values with the iteration index $k$, so that the optimization solution of the unconstrained new functio $F(\mathbf{x} ; \mathbf{a}(k), \mathbf{b}(k))$ converges to the optimization solution of the 
constrained objective function $f(\mathbf{x})$. The power parameter $p$ is a real number usually greater than 2 . In the PSCO method the new objective function is modified as follows:

$$
F\left(\mathbf{x} ; \mathbf{a}, \mathbf{b}, r_{a}(k)\right)=f(\mathbf{x})+\sum_{i=1}^{G} \frac{\mathbf{a}_{i}}{r_{a}(k)} \tilde{g}_{i}(\mathbf{x})+\sum_{j=1}^{H} \frac{\mathbf{b}_{j}}{r_{a}(k)}\left|h_{j}(\mathbf{x})\right|^{p}
$$

where this time the vector parameters $\mathbf{a}$ and $\mathbf{b}$ have fixed constant entries and the auxiliary functions $\tilde{g}_{i}(\mathbf{x})$ are defined as:

$$
\tilde{g}_{i}(\mathbf{x})=\left\{\begin{array}{ll}
\max \left[0, g_{i}(\mathbf{x})\right]^{\frac{1}{p}}, & g_{i}(\mathbf{x})<1 \\
\max \left[0, g_{i}(\mathbf{x})\right]^{p}, & g_{i}(\mathbf{x}) \geq 1
\end{array} \quad i=1, \ldots, G .\right.
$$

The auxiliary functions $\tilde{g}_{i}(\mathbf{x})$ are more strictly imposing the inequality constraints. As it can be seen the factors $\mathbf{a}_{i} / r_{a}(k)$ and $\mathbf{b}_{i} / r_{a}(k)$ increase with the iteration index $k$ approaching $\mathbf{a}_{i} / \epsilon$ and $\mathbf{b}_{i} / \epsilon$ at the end of the iterative process. In this way we provide a natural increase of the penalty term by integrating it in the already presented PSCO method for unconstrained problems. One advantage of the PSCO penalty function method over classical penalty function methods is that we can start from the beginning with very large values of the constants $\mathbf{a}$ and $\mathbf{b}$ without being plagued by numerical instability, as is the case with gradient based methods. Another advantage is that we are not constrained to start from the outside of the feasible region, the particles being initialized randomly inside the constraining box and moving in the feasible region during the process.

\section{Testing and Results}

Although the number of parameters of the PSCO method seems large, in reality there is no need to do too much tuning, some standard values giving almost always good results for a first run. For example the following set of parameter values almost always gives good results for a first run: $N_{\max }=400$, $N_{\text {min }}=30$, pow $=5.0, \alpha_{v}=0.95$. The Minkovski distance power parameter $p$ depends on the dimension of the search space $n$. For $n=2$ we can take $p=2$ and for $n>2$ a good choice is $p=\lfloor 2 n / 3\rfloor$. The precision parameter $\epsilon$ depends on the bounds of the constraining box and on our intuitive expectation for the size (diameter) of the basins of attraction of the modes. Usually the value 0.01 , i.e. $1 \%$ of each side length of the constraining box is a good starting choice. Depending on the results obtained for the particular problem after a first run and on the computing capabilities of the computer used, the 
parameters can be tuned for improved subsequent runs. For example, in our black box approach, we always start with a relative large number of particles because we always expect the worse, i.e. an objective function with a very large number of modes. If the optimization problem proves to be unimodal then a repeat of the run with much less particles (usually about 10 times less) will provide the same accurate results. But if the objective function proves to be highly multimodal then a repeat with a larger number of starting particles is necessary.

The method was tested on many known optimization test functions, unconstrained and constrained, unimodal and multimodal, but only the results for a testbed formed by the following functions are presented ([18], [19]):

De Jong's First Function (unimodal, $n=20, f_{1, \min }=0.0$ at $(0,0, \ldots, 0)$ ):

$$
\begin{aligned}
f_{1}(\mathbf{x}) & =\sum_{j=1}^{n} \mathbf{x}_{j}^{2} \\
& -10 \leq \mathbf{x}_{i} \leq 10, \quad i=1, \ldots, n
\end{aligned}
$$

Rastrigin's Function (multimodal, $n=10, f_{2, \min }=0.0$ at $\left.(0,0, \ldots, 0)\right)$ :

$$
\begin{aligned}
f_{2}(\mathbf{x}) & =10 n+\sum_{j=1}^{n}\left(\mathbf{x}_{j}^{2}-10 \cos \left(2 \pi \mathbf{x}_{j}\right)\right) \\
& -5.12 \leq \mathbf{x}_{i} \leq 5.12, \quad i=1, \ldots, n
\end{aligned}
$$

Ackley's Function (multimodal, $n=10, f_{3, \min }=0.0$ at $(0,0, \ldots, 0)$ ):

$$
\begin{aligned}
f_{3}(\mathbf{x}) & =20+e-20 e^{-0.2\left(\frac{1}{n} \sum_{j=1}^{n} \mathbf{x}_{j}^{2}\right)^{\frac{1}{2}}}-e^{\frac{1}{n} \sum_{j=1}^{n} \cos \left(2 \pi \mathbf{x}_{j}\right)} \\
& -30 \leq \mathbf{x}_{i} \leq 30, \quad i=1, \ldots, n
\end{aligned}
$$

Griewangk's Function (multimodal, $n=10, f_{4, \min }=0.0$ at $(0,0, \ldots, 0)$ ):

$$
\begin{aligned}
f_{4}(\mathbf{x}) & =\frac{1}{4000} \sum_{j=1}^{n} \mathbf{x}_{j}^{2}-\prod_{j=1}^{n} \cos \left(\frac{\mathbf{x}_{j}}{\sqrt{j}}\right)+1 \\
& -100 \leq \mathbf{x}_{i} \leq 100, \quad i=1, \ldots, n
\end{aligned}
$$

Salomon's Function (multimodal, $n=3, f_{5, \min }=0.0$ at $(0,0, \ldots, 0)$ ): 


$$
\begin{aligned}
f_{5}(\mathbf{x}) & =-\cos \left(2 \pi\left(\sum_{j=1}^{n} \mathbf{x}_{j}^{2}\right)^{\frac{1}{2}}\right)+0.1\left(\sum_{j=1}^{n} \mathbf{x}_{j}^{2}\right)^{\frac{1}{2}}+1 \\
& -10 \leq \mathbf{x}_{i} \leq 10, \quad i=1, \ldots, n
\end{aligned}
$$

Shubert's Function (multimodal, $n=2, f_{6, \min }=-186.7309$ at a total of 18 global minima):

$$
\begin{aligned}
f_{6}(\mathbf{x}) & =-\left(\sum_{i=1}^{5} i \cos \left((i+1) \mathbf{x}_{1}+1\right)\right)\left(\sum_{i=1}^{5} i \cos \left((i+1) \mathbf{x}_{2}+1\right)\right) \\
& -10.0 \leq \mathbf{x}_{1} \leq 10.0,-10.0 \leq \mathbf{x}_{2} \leq 10.0
\end{aligned}
$$

De Jong's Fifth Function (multimodal, $n=2, f_{7, \min }=0.9980$ at $(-31.9783,-31.9783))$ :

$$
\begin{aligned}
f_{7}(\mathbf{x}) & =\left(0.002+\sum_{i=-2}^{2} \sum_{j=-2}^{2}\left(5(i+2)+j+3+\left(\mathbf{x}_{1}-16 j\right)^{6}+\left(\mathbf{x}_{2}-16 j\right)^{6}\right)^{-1}\right)^{-1} \\
& -65.536 \leq \mathbf{x}_{1} \leq 65.536, \quad-65.536 \leq \mathbf{x}_{2} \leq 65.536
\end{aligned}
$$

and Optimal Reactor Design (multimodal, constrained, $n=8, f_{8, \min }=$ 3.9511):

$$
\begin{aligned}
& f_{8}(\mathbf{x})=0.4\left(\frac{\mathbf{x}_{1}}{\mathbf{x}_{7}}\right)^{0.67}+0.4\left(\frac{\mathbf{x}_{2}}{\mathbf{x}_{8}}\right)^{0.67}+10.0-\mathbf{x}_{1}-\mathbf{x}_{2} \\
& g_{1}(\mathbf{x})=0.0588 \mathbf{x}_{5} \mathbf{x}_{7}+0.1 \mathbf{x}_{1} \leq 1.0 \\
& g_{2}(\mathbf{x})=0.0588 \mathbf{x}_{6} \mathbf{x}_{8}+0.1 \mathbf{x}_{1}+0.1 \mathbf{x}_{2} \leq 1.0 \\
& g_{3}(\mathbf{x})=4.0\left(\frac{\mathbf{x}_{3}}{\mathbf{x}_{5}}\right)+\frac{2.0}{\mathbf{x}_{3}^{0.71} \mathbf{x}_{5}}+0.0588 \frac{\mathbf{x}_{7}}{\mathbf{x}_{3}^{1.3}} \leq 1.0 \\
& g_{4}(\mathbf{x})=4.0\left(\frac{\mathbf{x}_{4}}{\mathbf{x}_{6}}\right)+\frac{2.0}{\mathbf{x}_{4}^{0.71} \mathbf{x}_{6}}+0.0588 \frac{\mathbf{x}_{8}}{\mathbf{x}_{4}^{1.3}} \leq 1.0 \\
& 0.1 \leq \mathbf{x}_{i} \leq 10.0, \quad i=1, \ldots, 8
\end{aligned}
$$

In order to compare the results of different optimization methods from the computing efficiency perspective the $N F E$ (number of function evaluations) 
to convergence is used as a criterion. In our case, taking into account that our method is a parallel computing method, we can represent the computing process as a binary tree with the main execution thread starting to run at the root node of the binary tree. Each time a separation takes place the parent root continues to run with the first sub-swarm, while the second subswarm is handled by a new created child parallel execution thread. A path of execution is a path connecting the root node with a leaf node. In an ideal case in which we have a computer with a large number of processors (at least equal to the number of leaf nodes) a good efficiency criterion is the largest $N F E$ from the NFEs of the paths connecting the root node to the leaf nodes. Let us denote by $N F E^{\prime}$ this largest $N F E$ and by $N_{\text {paths }}$ the number of paths connecting the root node to the leaf nodes (which is the same as the number of parallel execution threads). Then we have:

$$
N F E^{\prime}=\max _{1 \leq i \leq N_{\text {paths }}} N F E_{i}
$$

Also, taking into account that the method determines more than one mode, we should average the $N F E^{\prime}$ to the number of determined modes. Let us denote by $N F E^{\prime \prime}$ the $N F E^{\prime}$ averaged to the number of modes (which is the same as $N_{\text {paths }}$ ), $N F E^{\prime \prime}=N F E^{\prime} / N_{\text {paths }}$. Table I below gives the computing results obtained for the functions considered in the testbed statistically analyzed for 10 runs. $S R$ denotes the Success Rate in identifying the cluster for the global optimum (in percentage), and $S D$ denotes the Standard Deviation. The common parameters were $N_{\max }=400, N_{\min }=30$, pow $=5.0$. The value of the $\epsilon$ parameter was 0.01 , with the exception of the Griewangk function for which (after tuning) it was 0.005 and the Salomon function for which (after tuning) it was 0.001 . The value of the $\alpha$ parameter was 0.95 with the exception of the Griewangk and Salomon functions for which (after tuning) it was 0.97. Considering that we take the black box approach, we expect large values for the $N F E^{\prime \prime}$ and subsequent runs with tuned parameters (for improved results) can produce even larger values for $N F E^{\prime \prime}$. For all the test functions, in cases of succes $(S R=100 \%)$ the global cluster was formed and the subsequent local optimization step was able to calculate the global mode with high accuracy.

An image with the final clusters projected on the first two dimensions for the Optimal Reactor Design problem is presented below in FIG. 1. 
Table 1: Testing Results

\begin{tabular}{|l|ccccc|}
\hline Test Function & $S R$ & $\overline{N F E}$ & $\overline{N F E^{\prime}}$ & $\overline{N F E^{\prime \prime}}$ & $S D\left(N F E^{\prime \prime}\right)$ \\
\hline First De Jong & $100 \%$ & 554823 & 554823 & 554823 & 0.0 \\
Rastrigin & $70 \%$ & 654578 & 231467 & 5172 & 689.50 \\
Ackley & $100 \%$ & 280360 & 280360 & 280360 & 0.0 \\
Griewangk & $100 \%$ & 987143 & 498751 & 12618 & 734.57 \\
Salomon & $80 \%$ & 4343523 & 169352 & 100 & 16.51 \\
Shubert & $100 \%$ & 95796 & 40916 & 1074 & 212.67 \\
Fifth De Jong & $100 \%$ & 91275 & 47817 & 1986 & 59.47 \\
Reactor Design & $100 \%$ & 1544911 & 584430 & 4989 & 897.09 \\
\hline
\end{tabular}

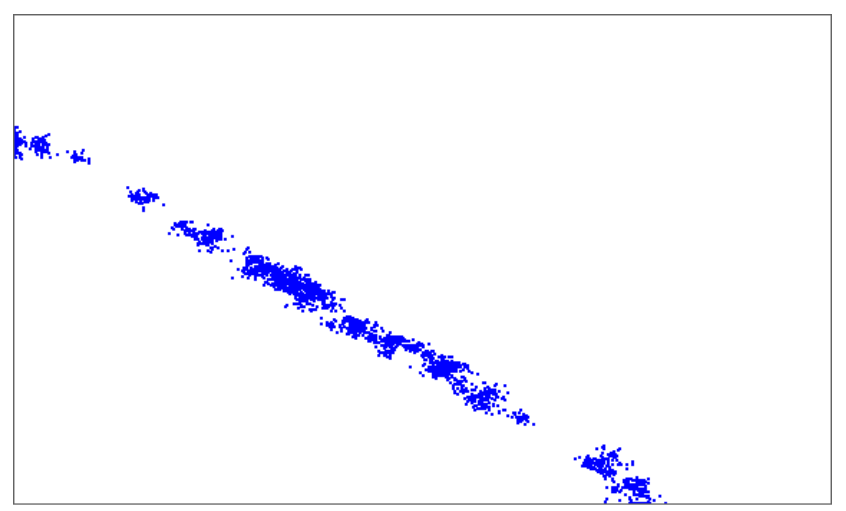

FIG. 1 - Optimal Reactor Design problem, final clusters

This image is a good example of the qualitative information the PSCO method can provide.

\section{Conclusions}

The paper presented a novel global optimization method based on combined ideas from Clustering Optimization and Swarm Intelligence, called Particle Swarm Clustering Optimization (PSCO). The method is simultaneously integrating the three steps of the classical Clustering Optimization methods by applying Swarm Intelligence techniques. In this way the computationally intensive Clustering Analysis phase is avoided. The PSCO method offers some advantages over other known Swarm Intelligence or Clustering Optimization methods. First the convergence of the method takes place in a rigorous controlled manner, providing the possibility to pre-calculate the number o iterations based on the required precision and the volumetric decrease rate, in this way being possible to estimate in advance the running time of the method. Knowing in advance the number of iterations helps in providing 
another advantage, the possibility to implement a rigorous sub-swarm size management strategy which allows the decrease or increase (as required) of the sub-swarm size with the volume of the search space. Another advantage of the PSCO method is the intrinsic separation of the initial swarm in independent sub-swarms, which can be independently handled (as optimization sub-problems) by parallel execution threads, in this way increasing the computational efficiency. The method was tested on many optimization test functions, unconstrained (box constrained) or constrained, with very good results.

\section{References}

[1] http://www.cs.sandia.gov/opt/survey/cluster.html, last access date: September 08, 2012

[2] M. Dorigo and T. Stützle, Ant Colony Optimization, MIT Press, Cambridge, Massachusetts, 2004

[3] J. Kennedy, R.C. Eberhart, and Y. Shi, Swarm Intelligence, Morgan Kaufmann, San Francisco, 2001

[4] J. Kennedy and R.C. Eberhart, Particle Swarm Optimization

[5] Y. Shi and R.C. Eberhart, A Modified Particle Swarm Optimizer

[6] M. Clerc, Particle Swarm Optimization, ISTE, London, U.K., 2006

[7] Xin-She Yang, Nature-Inspired Metaheuristic Algorithms, Luniver Press, Frome, U.K., 2008

[8] Xin-She Yang, Engineering Optimization - An Introduction with Metaheuristic Applications, John Wiley \& Sons, Hoboken, New Jersey, 2010

[9] Pablo Rabanal, Ismael Rodriguez, and Fernando Rubio, Using River Formation Dynamics to Design Heuristic Algorithms, Lecture Notes in Computer Science, 4618, (2007), 163-177

[10] E. Rashedi, H. Nezamabadi, and S. Saryazdi, GSA: A Gravitational Search Algorithm, Information Sciences, 179 (13), (2009), 2232-2248

[11] Hamed Shah-Hosseini, The intelligent water drops algorithm: a nature-inspired swarm-based optimization algorithm, International Journal of Bio-Inspired Computation (IJBIC), 1 (1/2), (2009), 71-79

[12] A. Kaveh and S. Talatahari, A Novel Heuristic Optimization Method: Charged System Search, Acta Mechanica, 213, (2010), 267-289

[13] J. D. Pintér, Global Optimization: Software, Test Problems, and Applications, Ch. 15 in Handbook of Global Optimization, Volume 2 (Ed. P. M. Pardalos and H. F. Romeijn), Kluwer Academic Publishers, Dordrecht, Boston, London, 2002 
[14] R. Horst and P.M. Pardalos, Handbook of Global Optimization, Kluwer Academic Publishers, Dordrecht, Boston, London, 1995

[15] P.M. Pardalos and M. Resende, Handbook of Applied Optimization, Oxford University Press, Oxford, U.K., 2002

[16] K. Veeramachaneni, T. Peram, C. Mohan, and L. Osadciw, Optimization using particle swarm with near neighbor interactions

[17] Li Xiaodong, A Multimodal Particle Swarm Optimizer based on Fitness Euclideandistance Ratio

[18] Floudas C., Handbook of Test Problems in Local and Global Optimization, Kluwer Academic Publishers, Dordrecht, Boston, London, 1999

[19] Z. Michalewicz, Genetic Algorithms + Data structures = Evolution Programs, Springer, Berlin, 1994

George Anescu

Power Plant Engineering Faculty

Polytechnic University of Bucharest

313 Splaiul Independentei, 060042

Bucharest

Romania

E-mail: george.anescu@gmail.com

Received: 15.11 .2012

Accepted: 28.01.2013 